\title{
Chladni figures in Andreev billiards
}

F. Libisch ${ }^{1}$, S. Rotter, and J. Burgdörfer

Institute for Theoretical Physics, Vienna University of Technology, Austria,

EU

We study wave functions and their nodal patterns in Andreev billiards consisting of a normal-conducting $(\mathrm{N})$ ballistic quantum dot in contact with a superconductor $(\mathrm{S})$. The bound states in such systems feature an electron and a hole component which are coherently coupled by the scattering of electrons into holes at the S-N interface. The wave function "lives" therefore on two sheets of configuration space, each of which features, in general, distinct nodal patterns. By comparing the wave functions and their nodal patterns for holes and electrons detailed tests of semiclassical predictions become possible. One

semiclassical theory based on ideal Andreev retroreflection predicts the electron- and hole eigenstates to perfectly mirror each other. We probe the limitations of validity of this model both in terms of the spectral density of the eigenstates and the shape of the wavefunctions in the electron and hole sheet.

We identify cases where the Chladni figures for the electrons and holes

drastically differ from each other and explain these discrepancies by limitations of the retroreflection picture.

\section{Introduction}

When Ernst Chladni first studied the formation of nodal patterns of standing waves two hundred years ago, the tools available to him where macroscopic objects like grains of sand on vibrating metal plates, and the underlying waves where acoustic waves. Electromagnetic waves were unequivocally established by Maxwell, Hertz, and others in the second half of the nineteenth century. In the beginnings of the twentieth century, de Broglie, and Schrödinger added matter waves within the framework of quantum mechanics to the list of wave-like phenomena. One immediate consequence of the ubiquity of wave-like behaviour of nature is the ubiquity of Chladni figures in a remarkably diverse array of physical phenomena and on very different length scales ranging from the macroscopic to the microscopic. In turn, the study of Chladni figures two hundred years after their first observation can still provide information on the underlying physical systems, for example on quantum dynamics and its semi-classical or short-wave limit.

We focus in the following on Chladni figures in microstructures which are the result of the wave-like behavior of the electronic dynamics and the presence of a superconducting interface. Technical advances in semiconductor science has made it possible to construct effectively two-dimensional micro- and nanodevices within which the electron propagation becomes phase coherent at sufficiently low temperatures[1]. Transport through such open "quantum billiards" featuring ballistic motion has become the focus of intensive research interest,

\footnotetext{
${ }^{1}$ florian at concord.itp.tuwien.ac.at
} 
both experimental $[2,3]$ and theoretical $[4,5,6]$. When the opening is replaced by a superconducting interface, the ballistic motion turns into bounded motion of an Andreev Billiard. As discovered by Andreev in 1964, the interface between a ballistic semiconductor $(\mathrm{N})$ and a superconducting metal (S) gives rise to the coherent scattering of electrons into holes[7]. Eigenstates of Andreev billiards thus feature an electron and a hole component coupled at the superconducting interface. Such a closed quantum billiard features a coupled two-sheeted configuration space, one sheet representing the motion of the electron, the other the motion of the hole. This two-sheeted configuration space translates into two coupled standing-wave patterns with their concomitant nodal lines or Chladni figures. In the present comunication we briefly discuss what can be learned from the nodal patterns of electron and hole components for the quantum dynamics of Andreev billiards and its semiclassical limit. The proverbial question referring to the acoustic analogue to billiards, "can you hear the shape of a drum", i.e. what are spectral signatures of the shape of the Dirichlet boundary, can be posed for the present system in different ways. "What are the spectral impacts of Andreev reflection at the S-N boundary?" or more provocatively, "Can you see Andreev reflection trajectories in the nodal patterns of the electron and hole wave?" We connect the nodal patterns found in the eigenstates of Andreev billiards to the periodic orbits predicted by semiclassical theory. We find that the similarity between electron and hole components of the eigenstates gives us a quantitative measure for the validity of a semiclassical Bohr-Sommerfeld approximation and its failure.

We investigate two distinct geometries: (i) the half-circle billiard, which features regular dynamics, and (ii) the mushroom billiard, whose counterpart with only Dirichlet boundary conditions features one regular island in a chaotic sea. Using the modular recursive Green's function method (MRGM) $[8,9]$ we calculate numerically not only the eigenenergies but also the corresponding quantum mechanical eigenstates of Andreev billiards. The analysis of the nodal patterns provides us with a direct quantitative measure for the degree of quantumclassical correspondence.

This report is organized as follows: In section 2, we briefly introduce the physics of Andreev reflection and methods for the quantum and semiclassical description of Andreev billiards. In section 3, we present results for eigenstates, in particular for the nodal patterns in the electron and hole wavefunctions. A short summary and outlook is given in section 4 .

\section{Andreev billiards}

\subsection{Andreev reflection}

The dispersion relation of an electron moving through a ballistic, normal conducting quantum dot is, in good approximation, a parabola [see Fig. 1(a)]. Thus, the electron can be treated as quasi-free particle with an effective mass $m_{\text {eff }}$ determined by the curvature of the parabola at the $\Gamma$ point $(k=0)$. The 
(a)

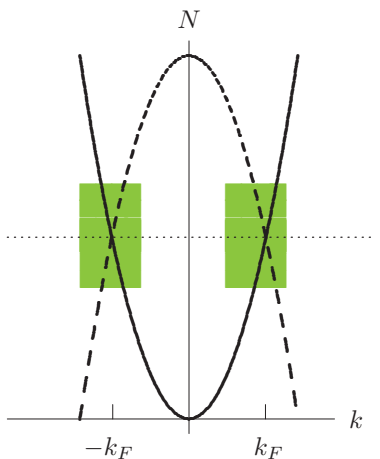

(c)

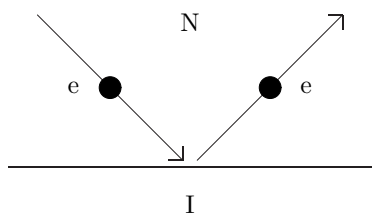

(b)

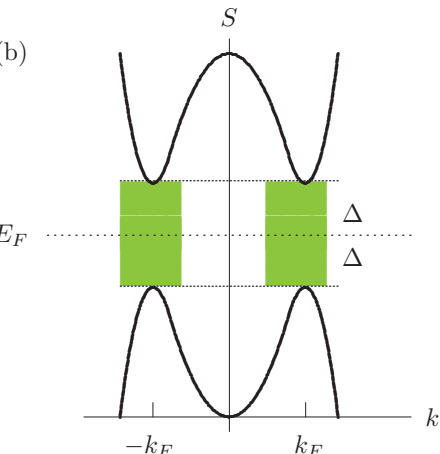

(d)

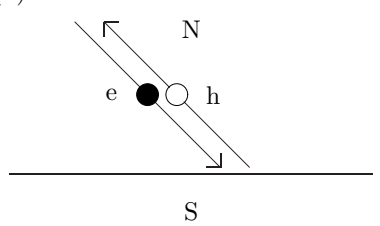

Figure 1: (a) Dispersion of a normal metal. Solid line: electron, dashed line: hole. In the absence of any interaction electron and hole lines cross. (b) Dispersion in the presence of the gap $\Delta$ in the superconductor near the Fermi edge. (c) Normal specular reflection of the electron at the interface described by Dirichlet boundary conditions. (d) Andreev retroreflection: electron-hole scattering at the S-N interface.

dispersion relation of a superconductor, on the other hand [see Fig. 1(b)], looks quite different[10]: A band gap of size $2 \Delta$ opens at the Fermi energy described by the theory of Bardeen, Cooper and Schrieffer (BCS) in terms of the formation of a condensate of paired electrons, so-called Cooper pairs, with binding energy of $\approx \Delta$, which represent the new ground state.

Consider an electron moving through a normal metal with a small excitation energy $\varepsilon<\Delta$ above the Fermi energy $E_{\mathrm{F}}$ [within the shaded area in Fig. 1(a)]. Once the particle hits a superconducting boundary, it is prohibited from entering the superconductor as a single electron, as there is no single electron state in the superconductor [see the shaded area in Fig. 1(b)]. However, the electron can enter the BCS ground state by forming a Cooper pair[11]. As a consequence, a second electron is taken from the energy $E_{\mathrm{F}}-\varepsilon$, creating a Cooper pair at the Fermi energy. This process leaves a hole in the Fermi sea, which is reflected back into the normal conductor. Under the assumption of the absence of other perturbing degrees of freedom (e.g. phonons) this process is phase coherent, i.e. there is a one-to-one relation between the phase of the electron and the Andreev reflected hole. Moreover, in contrast to specular reflection at an insulating boundary [see Fig. 1(c)], the hole is retroreflected back into the direction of the incident electron due to momentum conservation rather than specular reflected [see Fig. 1(d)]. 


\subsection{Classical dynamics: The role of periodic orbits}

The classical picture of retroreflection at the S-N boundary combined with electron-hole conversion has far-reaching consequences for the classical dynamics. Assuming exact, i.e. energy independent retroreflection, every trajectory in a closed billiard geometry with a single $\mathrm{S}-\mathrm{N}$ interface at its boundary [see Fig. 2(c)] becomes periodic in the extended phase space of electron $\left(\vec{r}_{e}, \vec{k}_{e}\right)$ and hole $\left(\vec{r}_{h}, \vec{k}_{h}\right)$. After two successive Andreev reflections the trajectory closes on itself. Unlike normal billiards, where the set of periodic orbits is of measure zero, they form continuous manifolds which, generally, fill the energy hypersurface, with exception of subsets of trajectories which do not touch the S-N interface. Moreover, a billiard featuring chaos for an entirely normal-conducting boundary is converted into a regular system governed by periodic motion when replacing part of its boundary by a superconductor.

A further consequence of retroreflection involves classical-quantum correspondence. To the extent that classical orbits provide, indeed, the skeleton of quantum wavefunctions the wavefunction for the electron should exactly mirror that of the hole, i.e. the two sheets of the wavefunction should be identical. The reason is that the classical trajectories in the electron and hole coordinates should have identical topologies and weights.

The assumption of exactly retracing electron-hole orbits (henceforth called the retracing approximation) provides a simple, yet reasonably accurate semiclassical description of Andreev billiards. Quantum mechanically, however, the (albeit small) energy difference between electron and hole will, in general, break the exact correspondence between electron and hole. Moreover, nondeterministic quantum mechanical effects such as quantum diffraction will lead to different nodal patterns for electron and hole. Turning the argument around, a disagreement between electron and hole wavefunction directly reveals quantum mechanical effects beyond the simple semiclassical approximation. By comparing the Chladni figures of electron and hole components of Andreev eigenstates, the validity of the retracing approximation can thus be directly assessed.
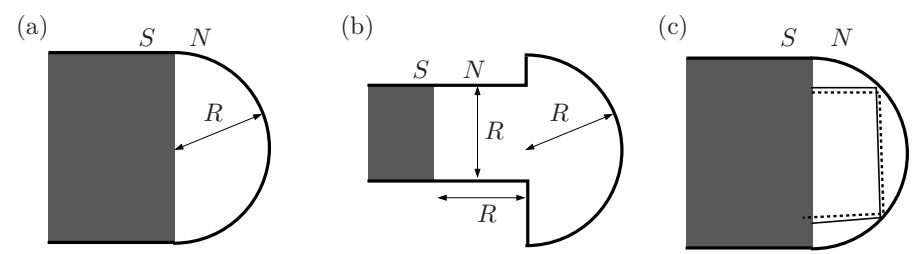

Figure 2: (a) Regular half-circle billiard with radius $R$ consisting of a superconducting (S) lead connected to a normal-conducting $(\mathrm{N})$ half-circle (superconducting area is shaded).(b) Mushroom billiard, the lead becomes superconducting after a distance $R$. (c) Periodic orbit created by two subsequent Andreev reflections. The path is traversed by the electron in one direction, and by the hole in the other. 


\subsection{Model system}

We consider quantum billiards for which the wavelength of electrons (or holes) is smaller but not neglegibly small compared to the system size. The wave equation used to model a superconductor-normal conductor hybrid system is the Bogolioubov-de Gennes (BdG) equation, a two-component extension of the Schrödinger equation

$$
\left(\begin{array}{cc}
H_{0} & \Delta \\
\Delta^{*} & -H_{0}^{*}
\end{array}\right)\left(\begin{array}{l}
u(\mathbf{x}) \\
v(\mathbf{x})
\end{array}\right)=\varepsilon\left(\begin{array}{l}
u(\mathbf{x}) \\
v(\mathbf{x})
\end{array}\right) .
$$

$H_{0}=p^{2} /\left(2 m_{\text {eff }}\right)+V(r)-E_{F}$ is the single particle Hamiltonian. $u(v)$ is the electron (hole) quasiparticle wavefunction, $\varepsilon$ the excitation energy of the electron (hole) above (below) the Fermi energy $E_{F}$. Because we want to investigate the bound states of a closed Andreev billiard, we consider an energy range $0 \leq \varepsilon \leq \Delta$, for which no propagating solutions of (1) in the superconducting region exist.

We investigate two distinct geometries, featuring both regular and chaotic motion in the absence of an S-N interface. A half-circular, ballistic normal conducting quantum dot of radius $R$ is attached at the straight side either to (i) a superconducting waveguide of width $2 R$, creating a half-circle billiard [see Fig. 2(a)], or (ii) to a waveguide of width $R$, which becomes superconducting after a distance $R$, creating a mushroom billiard [see Fig. 2(b)]. While the dynamics of the half-circle billiard is regular, the mushroom features mixed dynamics with exactly one regular island in the phase space of orbits contained in configuration space in the hat of the mushroom. As the superconductor is located at the base of the mushroom, only orbits outside this island, i.e. in the chaotic sea, visit the S-N interface and get converted into Andreev orbits.

As we consider only one superconducting lead, we can choose the superconducting gap parameter $\Delta$ to be real. We assume that the superconducting coherence length $\xi$ is small compared to any other scale of the system, in particular the wavelength and the size of the rectangular cavity, in order to use a step function model [12] for $\Delta=\left|\Delta_{0}\right| \theta\left(x_{\mathrm{S} N}-x\right)$. Our coordinate system is chosen such that the $\mathrm{S}-\mathrm{N}$ interface is located at $x_{\mathrm{S} N}=0$. For simplicity, we assume equal effective masses inside the $S$ and $N$ regions, required for an ideal interface between normal and superconductor. As a consequence, the probability for Andreev reflection in the energy range we consider is of order unity, up to corrections of order $\left(\Delta / E_{\mathrm{F}}\right)^{2}[11]$.

\subsection{Numerical technique}

We will give a short summary of the numerical techniques used to solve the BdG equation (1). A more detailed discussion can be found in Ref. [13].

In a first step, we calculate the scattering matrix $S(\varepsilon)$ of the open $\mathrm{N}$ cavity with the superconducting lead replaced by a normal conducting one. As this problem does not involve a superconductor, it can be treated using wellestablished techniques for conventional, ballistic quantum billiards. We use the 
modular recursive Green's function method (MRGM) [8, 9]. This method employs a tight binding grid discretization using symmetry-adapted coordinates (e.g. both cartesian and polar coordinates depending on the boundary) to assemble the Green's function of the structure. Both the scattering matrix $S$ and the wavefunction $\psi_{N}$ can then be calculated out of the Green's function.

Using the $S(\varepsilon)$ matrix, one can expand the wavefunction $\psi_{N}(x)$ as a superposition of incoming and outgoing transverse eigenstates $\chi_{n}(y)=\sqrt{2 / W} \sin (y n \pi / W)$ of the half-infinite waveguide as

$$
\psi_{N}=\sum_{n, m}\left(\begin{array}{c}
{\left[e^{+i k_{n}^{e} x}+e^{-i k_{n}^{e} x} S_{n m}(+\varepsilon)\right] c_{n}^{e}} \\
{\left[e^{-i k_{n}^{h} x}+e^{+i k_{n}^{h} x} S_{n m}(-\varepsilon)\right] c_{m}^{h}}
\end{array}\right) \chi_{n}(y)
$$

with $k_{n}^{e, h}=\sqrt{2\left(E_{\mathrm{F}} \pm \varepsilon\right)-k_{n y}^{2}}, k_{n}^{y}=n \pi / W$. In the $\mathrm{S}$ region, a similar expansion of $\psi_{S}(x)$ in terms of the analytic solutions of the $\mathrm{BdG}$ equation

$$
\psi_{S}=\sum_{n}\left[\left(\begin{array}{c}
e^{i \gamma} \\
1
\end{array}\right) b_{n}^{-} e^{-i q_{n} x}+\left(\begin{array}{c}
e^{-i \gamma} \\
1
\end{array}\right) b_{n}^{+} e^{i q_{n} x}\right] \chi_{n}(y)
$$

with $q^{ \pm}=\sqrt{2\left(E_{F} \mp i \sqrt{\Delta^{2}-\varepsilon^{2}}\right)-k_{y n}^{2}}$, and $\gamma=\arccos \frac{\varepsilon}{\Delta}$, is possible.

In the second step, we perform a wave function matching between the wavefunctions $\psi_{S}=\left(u_{S}, v_{S}\right)$ and $\psi_{N}=\left(u_{N}, v_{N}\right)$ in the $\mathrm{S}$ and $\mathrm{N}$ region of the $\mathrm{S}-\mathrm{N}$ interface, i.e. at $x_{\mathrm{S} N}[14]$. Matching conditions of the form

$$
\psi_{N}\left(x_{\mathrm{S} N}\right)=\psi_{S}\left(x_{\mathrm{S} N}\right),\left.\quad \partial_{x} \psi_{S}(x)\right|_{x=x_{\mathrm{SN}}}=\left.\partial_{x} \psi_{N}(x)\right|_{x=x_{\mathrm{S} N}}
$$

result in a set of linear equations. Eigenstates of the Andreev billiard can then be found by searching for zeros of the spectral determinant as a function of $\varepsilon[14]$. The present full quantum solution does not rely on any of the frequently involved approximations for Andreev systems such as the smallness of the gap $\Delta \ll E_{\mathrm{F}}$. It can therefore serve as benchmark for semiclassical approximations.

\section{Chladni figures}

\subsection{Half-circle billiard}

We are now ready to take a look at the Chladni figures created by electron and hole excitations inside an Andreev billiard. If the picture of Andreev reflections is valid [Fig.1(d)], we would expect a clear correspondence between electron and hole wavefunction due to the coupling at the S-N interface.

The eigenstates found in the regular half-circle billiard, indeed, fulfill these expectations featuring a near-perfect match between electron and hole eigenstate (see Fig. 3). We compare the nodal patterns of electron and hole for all eigenstates, and find very good agreement. Moreover, we observe a strong localization of the nodal patterns on a larger length scale. The wavefunction 

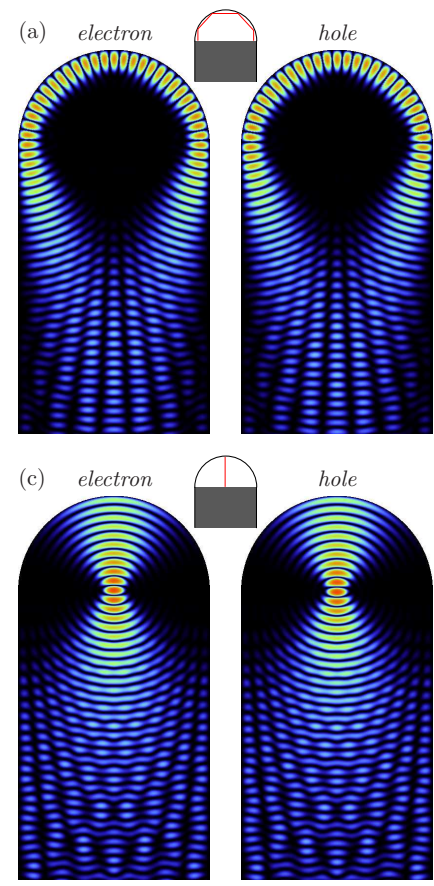

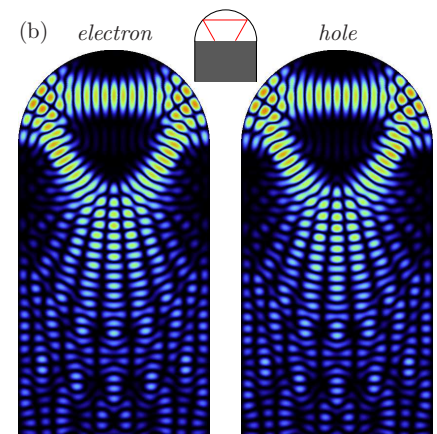

(d)

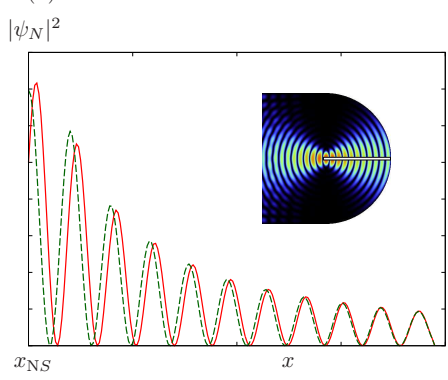

Figure 3: (a)-(c) Density of electron and hole eigenfunctions $|\psi|^{2}$ of the halfcircular Andreev billiard for three different excitation energies $\varepsilon=0.736 \Delta(\mathrm{a})$, $0.747 \Delta$ (b), and $0.836 \Delta(\mathrm{c})$. Red (white) corresponds to highest amplitude, black to lowest. The Fermi energy $E_{\mathrm{F}}=(21.5 \pi)^{2} / 2, \Delta=0.02 E_{\mathrm{F}}$. Both the similarity between electron and hole wavefunction and the enhancement along bundles of semiclassical orbits can be seen. (d) shows a cut through the electron (green, solid line) and hole (blue, dashed line) probability density depicted in (c) along the classical orbit shown in the inset.

displays a marked density enhancement along bundles of semiclassical periodic orbits (shown in the insets of Fig. 3)[15]. A similar phenomenon recently found in pseudointegrable normal billiards was called superscarring[16]. The coarsegrained density is enhanced along bundles of periodic Andreev orbits corresponding to different orbit lengths $l$ [see the insets in Fig. 3(a-c)]. States with lower eigenenergy $\varepsilon$ are associated with longer orbits. This observation can be understood by considering a Bohr-Sommerfeld quantization of periodic Andreev orbits discussed below.

\subsection{Semiclassical description}

The nodal patterns displayed in Fig. 3 can give direct insights into the process of the Bohr-Sommerfeld periodic orbit quantization. The total phase accumulated when traversing a periodic orbit should be an integer multiple of $2 \pi$. In contrast 
to a conventional one-component eigenstate, both the electron and hole part of the trajectory must be included. The phase the electron accumulates while traversing the billiard is subtracted from that of the hole retracing it carrying the opposite sign. However, due to the slight mismatch in energies $\left(E_{\mathrm{F}}+\varepsilon\right.$ for the electron, $E_{\mathrm{F}}-\varepsilon$ for the hole), a finite difference in phase between electron and Andreev reflected hole results. The phase difference is directly visualized by the nodal patterns along the classical periodic orbit in the electron and hole density [see Fig. 3(d)].

In addition, each Andreev reflection constitutes a phase $\gamma=\arccos \frac{\varepsilon}{\Delta}[17]$. Because one periodic orbit contains two Andreev reflections, this phase contribution has to be counted twice. The total phase accumulated over one periodic Andreev orbit $A$ is thus given by

$$
\frac{1}{\hbar} \oint_{A} p \mathrm{~d} q=l\left(k_{e}-k_{h}\right)=2(n \pi+\gamma)
$$

where $l$ is the length of $A$, and $k_{e, h}=\sqrt{2\left(E_{\mathrm{F}} \pm \varepsilon\right)}$ is the wavenumber of the electron or hole, respectively.

A Taylor expansion of Eq. (5) in terms of $\varepsilon$ yields a relationship between orbit length $l$ and excitation energy $\varepsilon$,

$$
l_{n}(\varepsilon)=(n \pi+\gamma) \frac{k_{\mathrm{F}}}{\varepsilon} \propto \frac{1}{\varepsilon} .
$$

Thus, the orbit length $l$ is, for fixed quantum number $n$, inversely proportional to the excitation energy of the particle $\varepsilon$. This explains the observed decrease in orbit length with increasing excitation energy. Note that the quantum number $n$ characterizes the quantized difference in action between the electron and the hole or equivalently, the phase shift in the nodal pattern between the two wavefunctions [see Fig. 3(d)].

Inserting into the left hand side of Eq. (6) the length of the classical periodic orbits visible as superscars in the eigenstates depicted in Fig. 3(a-c) predicts the corresponding eigenenergies with a high degree of accuracy. Thus, the eigenenergy of an Andreev state is not quantized by the number of oscillations along the orbit (proportional to $k_{\mathrm{F}}$ ) itself, as in conventional billiards, but by the difference in oscillations between two similar nodal patterns (proportional to $\left.k_{e}-k_{h}\right)$. Because $k_{e}-k_{h} \ll k_{e, h} \approx k_{\mathrm{F}}$, this quantity varies much more slowly with orbit length than in conventional billiards giving rise to the remarkable accuracy of semiclassical theories of Andreev billiards.

\subsection{State counting function}

We turn now to the large-scale energy spectrum of Andreev billiards. We insert the quantization condition (6) into a semiclassical expression of the state counting function $N$, i.e. the integrated density of states according to [18]

$$
N_{B S}(\varepsilon)=M \sum_{n=0}^{\infty} \int_{l_{n}(\varepsilon)}^{\infty} P(l) d l .
$$


The fact that within the retracing approximation, periodic orbits form continuous manifolds in Andreev billiards is incorporated in Eq. (7) via an integral over a continuous distribution of path lengths. All orbits of a length of at least $l=l_{n}(\varepsilon)$ contribute to the state counting function at energy $\varepsilon$. The weight of each orbit length $l$ is given by $P(l)$, the classical probability that an electron leaves the cavity after traversing a path of length $l$ [see Fig. 4(a)]. Note that $P(l)$ is the only geometry-specific information that enters the semiclassical prediction for the state counting function. The topology of individual orbits is irrelevant. All states share the same quantum number $n=0$ entering the quantization condition of Eq. (6), and are distinguished by the length of their periodic orbits. Their energies fall in a very narrow energy window, the lower bound of which is determined by the longest [Fig. 3(a)], and the upper bound by the shortest [Fig. 3(c)] classical path possible. Only a limited number of different path lengths are permitted in this geometry as shown in the classical path length distribution [see Fig. 4(a)].

The semiclassical prediction for $N(\varepsilon)$ according to Eq. (7) agrees with the quantum mechanical calculations of the state counting function

$$
N_{Q M}(\varepsilon)=\sum_{i} \theta\left(\varepsilon-\varepsilon_{i}\right)
$$

for the regular half-circle billiard remarkably well [see Fig. 4(b)]. It is now instructive to ask: Is such a good agreement to be expected? One key to the answer is the close similarity of nodal patterns and superscars in the electron and hole wavefunctions. The prerequisite for the validity of the semiclassical state-counting function (Eq. 7) is the retracing approximation. The validity of the latter implies that the wavefuntions on the two sheets closely mirror each other. For the half-circle billiard this is, indeed, the case (see Fig.3). Thus, the nodal patterns (or Chladni figures) and superscars can provide an immediate indication as to the validity of the semiclassical retracing approximation.

\subsection{Mushroom billiard}

If the diameter of the half-circle is chosen larger than the width of the superconducting lead, the billiard takes on the shape of a mushroom [see Fig. 2(c)]. In these structures, a wider variety of path topologies exists. As a consequence, the classical path length distribution shows pronounced structures [see Fig. 4(c)]. The state counting function now features several distinct cusps, corresponding to the different peaks in $P(s)$. These peaks correspond to topologically different bundles in phase space. As a consequence, we expect eigenstates associated with these different peaks to show different topological structures in their nodal patterns and superscars. Indeed, different classes of trajectory bundles can be distinguished by characteristically different nodal patterns: The first and most prominent peak in the pathlength spectrum $P(s)$ [marked (I) in Fig. 4(c)] denotes states with length $l \approx 4 R$ featuring regular nodal patterns similar to the states found in the half-circle billiard [see Fig. 5(b)]. The state counting function shows a corresponding cusp [marked (I) in Fig. 4(d)], which is predicted 
quite accurately by the semiclassical theory. A second cusp [marked (II) in Fig. 4(c,d)] features eigenstates reflecting once at the straight side of the mushroom hat [see Fig. 5(a)]. For both classes of states, patterns in the electron and hole wavefunctions agree very well.

The situation is qualitatively different, however, for eigenstates away from pronounced peaks of the path length distribution $P(s)$. An example is the relatively flat region of the state counting function where only few additional states are added [shaded in Fig. 4(d)]. The reason for the failure of the semiclassical retracing approximation can be readily seen in the wavefunctions: Electron and hole eigenstate do not mirror each other, most notably for the whispering gallery states, i.e. states trapped in the regular island of the mushroom[19, 20]. These states feature a strong asymetry in the overall probability density between electron and hole [see Fig.5(d)]. Clearly, such a situation is beyond the scope of the retracing approximation.

Another reason for the failure of retracing is diffractive scattering at the entrance corners to the mushroom. The Chladni figures show "fuzzy" patterns without discernable enhancement near a single periodic orbit. However, they do show high amplitudes at the diffractive corner. Furthermore, the patterns found for electron and hole do not agree [see Fig. 5(c)]. We performed numerical studies for different geometries with a varying number of diffractive corners to quantitatively understand the role of diffraction in Andreev billiards[21]. Our results show that quantum diffraction partly destroys the retracing properties of Andreev reflection.

\section{Conclusions}

We have studied the two-component wavefunctions of Andreev billiards for two different geometries with varying degree of classical-to-quantum correspondence. The nodal patterns (or Chladni figures) are shown to serve as a sensitive indication for the validity of the semiclassical approximations, in particular the retracing approximation.

For the regular half-circle billiard we find near-perfect agreement between the nodal patterns of electron and hole component of the eigenstate, indicating that the semiclassical retracing approximation is well suited to describe this geometry. Indeed, the periodic orbits predicted by semiclassical theory are directly mapped to strong enhancements of the nodal patterns along these orbits. This allows us to predict the eigenenergies of states by the lengths of the periodic orbits visible in their Chladni figures.

The mushroom Andreev billiard, whose normal-conducting counterpart features mixed dynamics, shows similar behaviour for those eigenstates dominated by short, broad bundles of classical trajectories. However, other eigenstates are not accounted for by the semiclassical approximation. For those states, electron and hole components show different nodal patterns. This is in line with the observed failure of the retracing approximation.

We can distinguish two distinct processes breaking the correspondence be- 
tween electron and hole eigenstate: (i) Coupling of one of the two components to the regular island located in the head of the mushroom, (ii) Diffractive effects at the corners of the mushroom. In summary, we have shown that Andreev billiards allow to determine the degree of quantum-classical correspondence by comparing the two nodal patterns of electron and hole component.

\section{Acknowledgements}

We would like to thank F. Aigner, J. Cserti, and A. Kormányos for valuable discussions. Support by the Austrian Science Foundation (Grant No. FWFP17359), and the British Council Vienna is gratefully acknowledged.

\section{References}

[1] D. Ferry, S. Goodwick, Transport in Nanostructures (Cambridge University Press, 1999)

[2] C.M. Marcus, A.J. Rimberg, R.M. Westervelt, P.F. Hopkins, A.C. Gossard, Phys. Rev. Lett. 69, 506 (1992)

[3] I.V. Zozoulenko, R. Schuster, K.F. Berggren, K. Ensslin, Phys. Rev. B 55, 10209 (1997)

[4] H.U. Baranger, A.D. Stone, Phys. Rev. B 40, 8169 (1989)

[5] E. Heller, Phys. Rev. Lett 53, 1515 (1984)

[6] O. Bohigas, M. Giannoni, C. Schmit, Phys. Rev. Lett. 52, 1 (1984)

[7] A. Andreev, Sov.Phys. JETP 19, 1228 (1964)

[8] S. Rotter, J.Z. Tang, L. Wirtz, J. Trost, J. Burgdörfer, Phys. Rev. B 62, $1950(2000)$

[9] S. Rotter, B. Weingartner, N. Rohringer, J. Burgdörfer, Phys. Rev. B 68, 165302 (2003)

[10] J. Bardeen, L. Cooper, J. Schrieffer, Phys. Rev. Lett. 108, 1175 (1957)

[11] G.E. Blonder, M. Tinkham, T.M. Klapwijk, Phys. Rev. B 25, 4515 (1982)

[12] C.W.J. Beenakker, Rev. Mod. Phys. 69, 731 (1997)

[13] F. Libisch, S. Rotter, J. Burgdörfer, A. Kormányos, J. Cserti, Phys. Rev. B 72, 075304 (2005)

[14] J. Cserti, A. Kormányos, Z. Kaufmann, J. Koltai, C.J. Lambert, Phys. Rev. Lett 89, 057001 (2002)

[15] L. Wirtz, J.Z. Tang, J. Burgdörfer, Phys. Rev. B 56, 7589 (1997) 
[16] E. Bogomolny, C. Schmit, Phys. Rev. Lett. 92, 244102 (2004)

[17] A. Kormányos, Z. Kaufmann, J. Cserti, C.J. Lambert, Phys. Rev. B 67, 172506 (2003)

[18] W. Ihra, M. Leadbeater, J. Vega, K. Richter, Eur. Phys. J. B 21, 425 (2001)

[19] E.G. Altmann, A. Motter, H. Kantz, Chaos 15, 033105 (2005)

[20] J. Cserti, A. Bodor, J. Koltai, G. Vattay, Phys. Rev. B 66, 064528 (2002)

[21] F. Libisch, S. Rotter, J. Burgdörfer, Phys.Rev.B 73, 045324 (2006) 


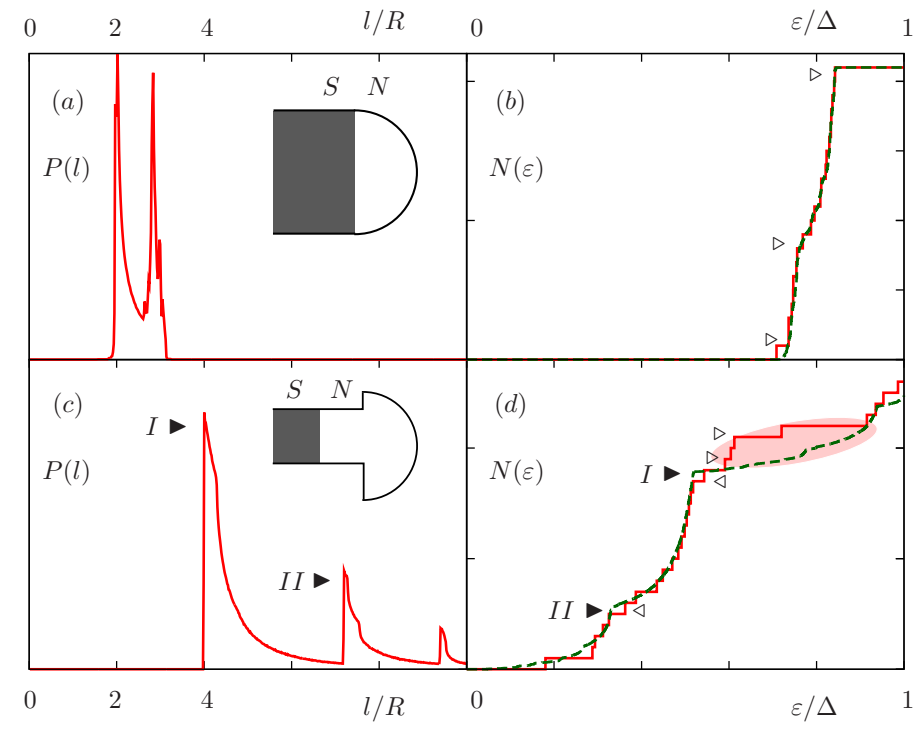

Figure 4: (a) Path length distribution $P(l)$ and (b) quantum mechanical (red staircase) and semiclassical (green dashed line) state counting function of the half-circle Andreev billiard. The Fermi energy $E_{\mathrm{F}}=(21.5 \pi)^{2} / 2, \Delta=0.02 E_{\mathrm{F}}$. Eigenstates are shown in Fig. 3 for energies marked by open triangles. (c) Path length distribution $P(l)$ and (d) state counting function $N(\varepsilon)$ of the mushroom Andreev billiard. The Fermi energy $E_{\mathrm{F}}=(17.5 \pi)^{2} / 2, \Delta=0.02 E_{\mathrm{F}}$. Two peaks in the path length distribution [marked $I$ and $I I$ in (c)] result in corresponding cusps in the state counting function [marked accordingly in (d)]. Open triangles denote energies for which eigenstates are shown in Fig. 5. 

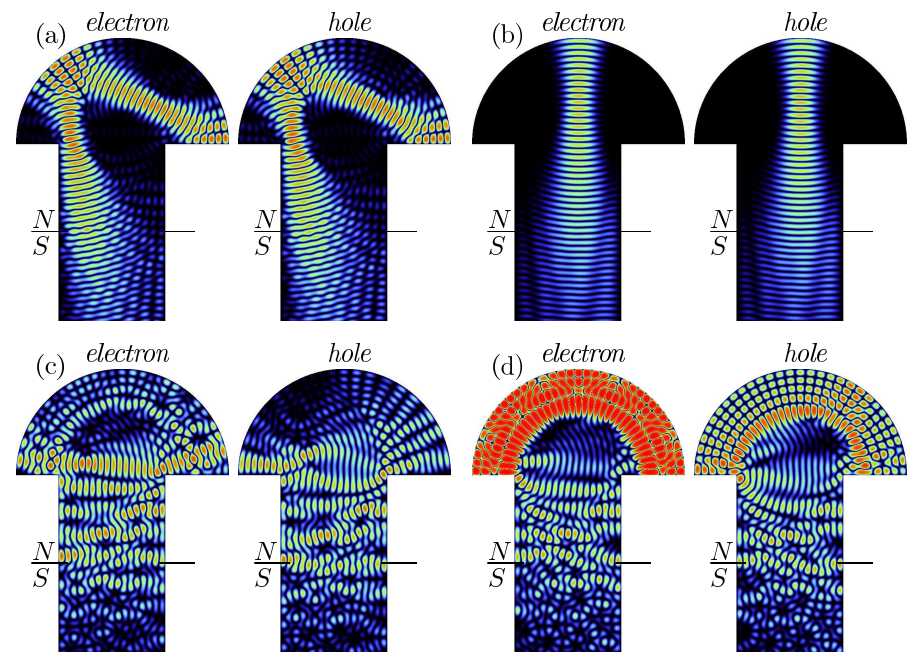

Figure 5: (a)-(d) Density of electron and hole eigenfunctions $|\psi|^{2}$ of the mushroom Andreev billiard for three different excitation energies $\varepsilon=0.33 \Delta(\mathrm{a})$, $0.52 \Delta(\mathrm{b}), 0.59 \Delta(\mathrm{c})$, and $0.61 \Delta(\mathrm{d})$. The Fermi energy was chosen at $E_{\mathrm{F}}=$ $(17.5 \pi)^{2} / 2, \Delta=0.02 E_{\mathrm{F}}$. The corresponding state counting function is shown in Fig. 4. 